\title{
Optimal solution to the fractional knapsack problem for LTE overload-state scheduling
}

\begin{abstract}
The growing variety and consumption of the mobile services throughout the Long Term Evolution (LTE) mobile networks lead to various challenging issues in radio resource scheduling. One of the main challenges in LTE resource management is how to allocate the scare available bandwidth, especially in overload states of the network, to the services which have different characteristics and quality requirements. In this paper, we study the optimal solution to LTE downlink resource allocation problem which has already been cast as the fractional knapsack optimization problem. The performance of this solution is investigated by a wide range of demands from different classes of services, over sequences of alternating overload and normal states of the network. The simulation results demonstrate that the proposed method supplies diverse quality requirements of different service classes in compromise with data rate enhancement. System performance has been evaluated in terms of loss, delay and throughput in comparison with reference algorithms.
\end{abstract}

Keyword: LTE networks; Downlink resource allocation; Fractional knapsack problem; Overload-state 